\title{
Fatores de risco e proteção à saúde mental infantil: o contexto familiar
}

\author{
Risk and protective factors for mental health in \\ children: the family context
}

\author{
Thelma Simões Matsukura' ${ }^{1}$, Amanda Dourado Souza Akahosi \\ Fernandes ${ }^{2}$, Maria Fernanda Barboza Cid $^{3}$
}

\begin{abstract}
MATSUKURA, T. S.; FERNANDES, A. D. S. A.; CID, M. F. B. Fatores de risco e proteção à saúde mental infantil: o contexto familiar. Rev. Ter. Ocup. Univ. São Paulo, v. 23, n. 2, p. 122129, maio/ago. 2012.

RESUMO. Algumas características familiares podem trazer riscos para o desenvolvimento saudável da criança, dentre elas se destacam a baixa renda, baixa escolaridade dos pais, altos níveis de estresse familiar. Em conjunto aos fatores de risco a que estão submetidas às crianças, tem-se os fatores de proteção, que podem atuar favorecendo o desenvolvimento humano na presença do risco. O objetivo do presente estudo foi identificar associações entre saúde mental infantil e variáveis consideradas de risco e proteção presentes no contexto familiar de crianças do ensino fundamental. A pesquisa foi realizada junto a 39 responsáveis por crianças do $1^{\circ}$ ao $5^{\circ}$ ano do ensino fundamental I, de uma escola pública, localizada na periferia da cidade de São Carlos-SP. Foram utilizados os instrumentos: Questionário de Capacidades e Dificuldades (SDQ), Critério de Classificação Econômica Brasil (CCEB) e o Questionário de Atividades Cotidianas (QAC). Foram realizadas análises estatísticas descritivas, de correlação e de comparação entre grupos, sendo que para as últimas foram utilizados os testes não paramétricos de Kendall's e Mann-Whitney. Os resultados obtidos mostraram que a variável dar broncas sem motivos e responsável não brincar com a criança está relacionada com a presença de problemas de saúde mental. Em contrapartida, a variável ter regras e responsabilidades na família foi relacionada a melhores níveis de saúde mental na criança.
\end{abstract}

DESCRITORES: Saúde mental; Criança; Família/psicologia; Fatores de risco; Psicologia da criança/recursos humanos.

\footnotetext{
1. Professora Associada do Departamento de Terapia Ocupacional - Universidade Federal de São Carlos (UFSCar).

2. Mestranda em Terapia Ocupacional - Universidade Federal de São Carlos (UFSCar).

3. Professora Assistente do Departamento de Terapia Ocupacional da Universidade Federal de São Carlos (UFSCar).

Endereço para correspondência: Departamento de Terapia Ocupacional da UFScar. Rodovia Washington Luís, Km 235, Monjolinho.
} 


\section{INTRODUÇÃO}

saúde mental pode ser compreendida como
“...) um estado de bem-estar no qual o
indivíduo percebe as suas capacidades, pode lidar com o stress normal da vida, pode trabalhar de forma produtiva e frutífera e é capaz de contribuir positivamente para sua comunidade" (OMS; 2001, p. 1).

No que se refere à criança e ao adolescente, autores têm apontado que aspectos presentes no ambiente em que vivem são os mais diretamente ligados à presença ou ausência de problemas relativos à saúde mental infantil (ASSIS et al., 2009; FERRIOLLI et al., 2007; PACHECO et al., 2005; HALPERN; FIGUEIRAS, 2004).

As características que são apontadas enquanto fatores de risco para a saúde mental infantil, quando focaliza-se o contexto familiar, englobam, dentre outras: baixa renda, baixa escolaridade dos pais, altos níveis de estresse familiar, baixos níveis de suporte social, altos níveis de discórdia marital, depressão e doença psiquiátrica dos pais (FLEITLICH; GOODMAN, 2000; HALPERN; FIGUEIRAS, 2004). Segundo Coie et al. (1993) riscos são variáveis associadas a uma alta probabilidade de desenvolver nos indivíduos a ele expostos, problemas de saúde mental, em diferentes níveis de severidade.

Conjuntamente aos fatores de risco aos quais estão submetidas às crianças, tem-se os fatores de proteção, que podem ser entendidos como recursos pessoais ou sociais que amenizam ou inibem o impacto do risco (GREENBERG et al., 2001). Os fatores de proteção podem atuar favorecendo o desenvolvimento humano, quando este está sendo ameaçado pela exposição ao risco e podem ser identificados e ativados na situação de risco (COIE et al., 1993; DEKOVIC, 1999; GREENBERG et al., 2001).

Quanto aos fatores protetivos, Mondin (2005), relata que alguns aspectos existentes na família podem colaborar para o desenvolvimento emocional, na presença do risco, como por exemplo, redes de apoio social e relações mais participativas e democráticas na família.

Estudos epidemiológicos que investigam problemas de saúde mental em crianças e adolescentes no mundo, apontam para uma grande variação nas taxas de prevalência encontradas (de aproximadamente de $10 \%$ a $50 \%$ ), no entanto, os dados permitem estimar que uma entre quatro a cinco crianças e adolescentes no mundo sofre de algum transtorno mental (PATEL et al., 2007; ASSIS et al., 2009).

No Brasil, em estudo de revisão sobre pesquisas desenvolvidas no período de 1980 a 2009, Paula et al. (2010) encontraram 13 estudos, que identificaram taxas de prevalência de problemas de saúde mental infantil com variações entre 12 a $25 \%$, ou seja, as taxas encontradas em estudos nacionais e internacionais são semelhantes.

Estudos nacionais investigaram aspectos relacionados à saúde mental infantil, tanto do ponto de vista epidemiológico, quanto em relação aos fatores que podem ser associados/correlacionados com a presença e/ ou ausência de problemas emocionais em crianças, tais como os fatores de risco e proteção presentes nas esferas individual, familiar e extra-familiar (FERRIOLI et al., 2007; PUNTEL, 2005; FLEITLICH; GOODMAN, 2000; CID; MATSUKURA, 2010).

Puntel (2005), desenvolveu estudo com 100 crianças de 6 a 12 anos de idade, usuários de um Núcleo de Atenção Primaria do Programa de Atenção Primaria e Saúde da Família no município de Ribeirão Preto-SP, que objetivou identificar riscos para transtornos emocionais. A autora encontrou associações entre a presença de transtornos emocionais nas crianças e a presença de situações adversas nos contextos familiar e escolar e também de dificuldades escolares.

Vitolo et al., (2005), realizaram estudo com 454 crianças em Taubaté-SP, cujo objetivo era verificar a prevalência e os fatores de risco para problemas de saúde mental infantil e a relação com as crenças e atitudes educativas dos cuidadores. Foi constatado relações entre punição física grave, problemas de saúde mental dos cuidadores, condição socioeconômica da família e problemas de saúde mental nas crianças.

Cid e Matsukura (2010) desenvolveram um estudo exploratório comparativo, com o objetivo de identificar os estilos parentais e os níveis de suporte social de mães com transtorno de humor e correlações com níveis de desenvolvimento emocional de seus filhos. Os resultados indicaram de que as mães com transtorno de humor são menos satisfeitas com o suporte social que recebem, punem mais de forma inconsistente seus filhos que as mães do grupo controle e possuem estilo parental considerado de risco. Quanto ao desenvolvimento emocional dos filhos, os resultados não mostraram diferenças significativas entre os grupos, porém, identificou-se uma associação entre estilo parental de risco e comportamentos antissociais nas crianças. As autoras apontam que estudos envolvendo amostras mais amplas e representativas devem ser implementados para aprofundar a avaliação dessas relações inicialmente identificadas.

O objetivo do presente estudo é identificar possíveis relações entre saúde mental infantil e situação sócioeconômica da família, contexto familiar e práticas de cuidado dos pais em relação às crianças. Dessa forma, visa contribuir com esse campo de estudos, na medida em que 
investiga os fatores do ambiente familiar que podem estar relacionados à manifestação de problemas de saúde mental em crianças. Além disso, acredita-se que possa fornecer subsídios para o planejamento e implementação de serviços e políticas públicas no campo da saúde mental infantil.

Observa-se que o estudo foi realizado em uma escola municipal da cidade de São Carlos-SP, no ano de 2009.

\section{METODOLOGIA}

\section{Participantes}

Participaram desta pesquisa 39 responsáveis por crianças estudantes do primeiro ao quinto ano do ensino fundamental I de uma escola pública municipal da cidade de São Carlos-SP.

Dentre os responsáveis, $84,6 \%$ eram mães biológicas, $53,8 \%$ possuíam entre $26-35$ anos e a maioria não completou o ensino fundamental.

Quanto as crianças, $74,4 \%$ eram meninas e a média das idade foi 8,64 anos. Em relação ao ano em que estudam, $51,2 \%$ cursam o terceiro e quarto ano do ensino fundamental.

Observou-se que $76,9 \%$ das famílias recebem somente um salário mínimo e a maioria são provenientes da classe social D, seguidos da C1, C2.

\section{Local}

O estudo foi realizado em São Carlos-SP em uma área periférica, designada como "zona crítica", na qual possui os maiores índices de vulnerabilidade social, destacando a extrema pobreza, altos índices de violência, desemprego, tráfico e consumo de drogas, prostituição infantil, baixa escolaridade da população (CAMPOS et al., 2003).

A região se caracteriza pela ocupação irregular acarretando riscos à população uma vez que se localiza próxima a uma área de risco e preservação ambiental denominada de "buracão". Observa-se também escassez de serviços públicos de educação, saúde esporte e lazer (CAMPOS et al., 2003).

\section{Procedimentos}

Foram aplicados os seguintes instrumentos:

Critério de classificação econômica Brasil (CCEB)

O CCEB- comumente tratado como Critério Brasil calcula o poder de compra dos indivíduos e famílias urbanas, classificando-os por classes econômicas ao invés do critério de classes sociais. A versão utilizada nesta pesquisa é do ano de 2009, tendo como base o LSE- levantamento econômico de 2006 e 2007 (ABEP-Associação Brasileira de Empresas de Pesquisa, 2009). A aplicação deste instrumento teve como objetivo avaliar a classificação econômica dos participantes.

Questionário de Capacidades e Dificuldades (SDQ)

O SDQ foi utilizado para detectar problemas de saúde mental infantil. É composto por 25 itens, subdivididos em 5 sub-escalas que avaliam: hiperatividade, sintomas emocionais, problemas de conduta, relações interpessoais e comportamento pró-social. Observa-se que no presente estudo, foi utilizada a versão para pais. Este questionário tem sido traduzido e validado para mais de quarenta países, apresentando tradução para o português (CURY; GOLFETO, 2003; FLEITLICH; GOODMAN, 2000).

\section{Questionário de Atividades Cotidianas (QAC)}

Este questionário é composto por 42 questões abertas e fechadas, que abordaram aspectos referentes à identificação geral da criança, situação familiar, escolaridade dos pais e responsáveis, vivência de atividades de lazer pela criança e família e do cotidiano de atividades conjuntas entre pais e filhos (CID; MATSUKURA, 2009).

\section{Procedimentos Éticos}

O projeto de pesquisa foi submetido ao Comitê de Ética em Pesquisa em Seres Humanos da UFSCar. Após a sua aprovação, expressa pelo processo n 23112.004819/20087 , iniciou-se os procedimentos para a coleta de dados, que serão detalhados a seguir.

\section{Seleção dos participantes}

Selecionada a escola, localizada na periferia e identificado o número total de participantes por meio de procedimento estatístico de composição de amostra, foi calculado o número de participantes para cada série $\left(1^{\circ}\right.$. ano, $2^{\circ}$. ano, etc.). Os alunos foram sorteados. Foram utilizados números em ordem crescente, de forma que, para a seleção, buscou-se o valor sorteado referente à ordem dos nomes dos alunos nas listas de chamada de cada classe. Por exemplo, o número 14 do primeiro ano $\mathrm{A}$ da escola foi sorteado. Para identificá-lo, checava-se na lista de chamada desta série, qual criança correspondia ao número 14. Ressalta-se que além da amostra "principal" calculada e sorteada, foi composta, também, uma amostra de substituição, para os casos de impossibilidade de realização da coleta de dados com aqueles da primeira amostra.

Após a identificação das crianças, foi dirigida cartaconvite, enviada pelas professoras das crianças, para os pais de todos os alunos sorteados informando sobre o estudo e sobre o fato de que a pesquisadora entraria em contato por 
telefone ou diretamente, na residência, para convidá-los pessoalmente para a participação no estudo.

Após o envio das cartas foi iniciado o contato com os responsáveis. Os objetivos do estudo foram novamente explicados. Para aqueles que aceitaram participar, realizouse um encontro para a assinatura do Termo de Consentimento Livre e Esclarecido. Feito isso, os instrumentos de coleta de dados foram apresentados na forma de entrevistas.

\section{Coleta de dados}

Houve até três tentativas de contato em dias, horários e de formas diferentes com os participantes que não eram encontrados. Aqueles que não aceitaram participar ou que o contato não foi possível nas três tentativas eram substituídos por outros que compunham a amostra reserva.

\section{Análise dos dados}

Os resultados advindos da aplicação dos instrumentos padronizados foram analisados de acordo com planilhas de cálculos, a partir dos quais as análises estatísticas descritivas foram realizadas.

Para as análises estatísticas de correlação utilizou- se o teste Kendall's e para as analises de comparação entre grupos, utilizou-se o teste não paramétrico Mann-Whitney. Observa-se que os testes apresentavam resultado significante quando o valor da estatística que o descreve ( $p$-valor) fosse menor do que 0,05 , admitindo-se como probabilidade de erro, o valor de $5 \%$.

\section{RESULTADOS}

\section{Principais resultados descritivos}

Neste tópico, apresentam-se os principais resultados descritivos obtidos no presente estudo.

Em relação a saúde mental das crianças estudadas, avaliada pelos responsáveis por meio do SDQ, os resultados serão apresentados na Tabela 1- Questionário de Capacidades e Dificuldades (SDQ).

Observa-se na Tabela 1 que a maioria $(58,9 \%)$ dos responsáveis avaliam que as crianças apresentam um total de dificuldades sócio-emocionais considerado clínico, ou seja, que apresentam problemas de saúde mental.

Tabela 1 - Questionário de Capacidades e Dificuldades (SDQ)

\begin{tabular}{l|ccc}
\hline & Saudável N(\%) & Limítrofe N(\%) & Clínico N(\%) \\
\hline Escalas de sintomas emocionais & $15(38,46)$ & $2(5,12)$ & $22(56,41)$ \\
Escala de problemas de conduta & $9(23,07)$ & $9(23,07)$ & $21(53,8)$, \\
Escala de hiperatividade & $20(51,28)$ & $7(17,94)$ & $12(30,76)$ \\
Escala de problemas de & $13(33,33)$ & $7(17,94)$ & $19(48,71)$ \\
relacionamento & $\mathbf{1 2 ( 3 0 , 8 )}$ & $\mathbf{4 ( 1 0 , 2 5 )}$ & $\mathbf{2 3}(\mathbf{5 8 , 9 )}$ \\
Total de dificuldades & $31(79,4)$ & $3(7,6)$ & $5(12,8)$ \\
Suplemento de impacto & & & 5 \\
\hline
\end{tabular}

Quanto às escalas, as crianças apresentaram principalmente dificuldades em relação aos sintomas emocionais, seguidos dos problemas de conduta, problemas de relacionamento com colegas e hiperatividade.

Dentre os resultados advindos do QAC avaliou-se que $53,8 \%$ das crianças têm atividades de lazer.

Quanto às práticas cotidianas de cuidado dos pais em relação às crianças, $20,5 \%$ dos participantes relatam dar bronca na criança mesmo quando não há motivo. A grande maioria relata fazer carinho $(76,9 \%)$, conversar sobre assuntos da escola $(97,4 \%)$ e brincar com as crianças $(74,4 \%)$.

Em relação a existência de uma rotina de atividades que a criança deve seguir em casa, 71,8\% tem uma rotina estabelecida. Já em relação à existência de regras e responsabilidades que todos os membros da família conhecem e devem cumprir, $43,5 \%$ dos responsáveis relatam que possuem tal prática.
Em relação aos conflitos existentes no ambiente familiar, 35,8\% dos responsáveis apontam que há brigas em casa e, destas, a maioria são discussões $(71,4 \%)$. Quanto aos atores envolvidos nas brigas e discussões os filhos (discussões entre os irmãos) receberam maior percentagem.

\section{Resultados das relações encontradas entre algumas variáveis do QAC e a Saúde Mental das crianças}

Foi realizado o teste de Kendall's para correlacionar as sub-escalas do SDQ às variáveis: renda familiar, desempenho da criança na escola segundo percepção do responsável, número de pessoas que residem na casa, idade e escolaridade do responsável. No entanto, as correlações estatísticamente significativas encontradas, tiveram índice menor que 0,3 o que indica uma correlação muito fraca e, portanto, optou-se por não considerá-las, ou seja, o estudo não encontrou correlações consideradas estatísticamente 
significativas entre tais variáveis.

\section{Resultados das análises comparativas}

Para as análises comparativas entre os grupos foi utilizado o teste estatístico de Mann Whitney, de forma que os escores advindos do SDQ foram comparados entre o grupo de responsáveis que relatou "sim" e o grupo de responsáveis que relatou "não" para as variáveis investigadas.

Apresenta-se na Tabela 2 - Resultados das análises de comparação entre grupos no que se refere ao SDQ, resultados estatisticamente significativos encontrados.

Tabela 2 - Resultados das análises de comparação entre grupos no que se refere ao SDQ

\begin{tabular}{|c|c|c|c|c|c|c|c|c|c|c|c|c|c|}
\hline & \multicolumn{3}{|c|}{ Regras e responsabilidades } & \multicolumn{3}{|c|}{ Criança repetiu de ano } & \multicolumn{4}{|c|}{$\begin{array}{l}\text { Responsável dá } \\
\text { broncas sem } \\
\text { motivo }\end{array}$} & \multicolumn{3}{|c|}{ Responsável Brinca } \\
\hline & Sim & Não & $\mathbf{z}$ & Sim & Não & $\mathbf{z}$ & Sim & $\mathbf{N}$ & & $\mathbf{z}$ & Sim & Nã & $\mathbf{z}$ \\
\hline Sintomas emocionais & - & - & - & - & - & - & - & - & & - & - & - & - \\
\hline $\begin{array}{l}\text { Problemas de } \\
\text { relacionamento }\end{array}$ & - & - & - & - & - & - & 4,2 & 3,1 & & $02 *$ & - & - & - \\
\hline $\begin{array}{l}\text { Hiperatividade/ defic. } \\
\text { atenção }\end{array}$ & - & - & - & - & - & - & & & & - & 4,06 & 7,3 & $2,7^{*}$ \\
\hline Problemas de conduta & 3,11 & 4,5 & $2,15^{*}$ & - & - & - & & & - & - & - & - & - \\
\hline Total & - & - & - & - & - & - & & & - & - & - & - & - \\
\hline $\begin{array}{l}\text { Comportamento pró- } \\
\text { social }\end{array}$ & - & - & - & 7,1 & 8,6 & $2,01 *$ & & & & - & - & - & - \\
\hline
\end{tabular}

Os resultados apresentados na Tabela 2 apontam que as crianças apresentam mais problemas de hiperatividade quando os responsáveis relatam não brincar com a criança e mais problemas de relacionamento quando os responsáveis relatam que dão broncas sem motivos.

Em contrapartida, quando o responsável relata que na família da criança existem regras e responsabilidades que todos conhecem e cumprem, as crianças apresentam menos problemas de conduta. Outro resultado se refere à reprovação escolar, ou seja, as crianças que nunca repetiram de ano, apresentam mais comportamentos pró-sociais que aquelas que já repetiram.

Aponta-se que as variáveis: - "o responsável faz carinho na criança", "a criança tem amigos", "os pais da criança vivem juntos", "atividades de lazer da criança e da família", "existência de brigas na família", "responsável trabalha" - também foram analisadas, mas não foram encontradas diferenças significativas entre os grupos.

\section{DISCUSSÃO}

O objetivo do estudo foi identificar e aprofundar a investigação de possíveis relações entre saúde mental infantil e situação sócio-econômica da família, contexto familiar e práticas de cuidado dos pais em relação às crianças.

Em relação aos problemas de saúde mental infantil, o estudo revelou um alto número de casos clínicos para o escore geral do SDQ, $58,9 \%$ de crianças, sendo que se considerarmos os casos limítrofes, $69,15 \%$ de crianças apresentaram problemas na esfera da saúde mental ou estão em alto risco para o desenvolvimento de problemas.

Este achado é bastante superior às taxas encontradas em estudos de prevalência realizados no Brasil e muito superior aos índices encontrados em estudos internacionais (PAULA et al., 2006; VITOLO et al., 2005).

Uma hipótese para este resultado refere-se ao fato de as crianças serem provenientes de uma escola situada em um bairro bastante vulnerável do município. Fleitlich e Goodman (2001), desenvolveram um estudo de prevalência, utilizando o SDQ, com crianças e adolescentes provenientes de três áreas distintas da cidade Campos do Jordão - SP (uma favela, uma comunidade urbana bem estabelecida e uma comunidade rural). Os autores analisaram as taxas encontradas abarcando todos os grupos (15\%) e também de forma separada e encontraram que as crianças e adolescentes residentes da favela apresentaram mais problemas relativos à 
saúde mental (22\%) que aqueles residentes das outras áreas. Paula et al. (2007) também identificaram a prevalência de problemas de saúde mental em crianças e adolescentes de comunidade urbana de baixa renda do município de EmbuSP, utilizando amostra probabilística de conglomerados incluindo todos os domicílios elegíveis. Foi encontrada taxa de prevalência para problemas de saúde mental em crianças e adolescentes de $24,6 \%$, ou seja, a situação socioeconômica, especificamente a renda e o pertencimento a regiões mais desfavorecidas é uma variável que parece estar relacionada à saúde mental das crianças e tem sido apontada pelos estudos como um importante fator de risco e foi avaliada pelo presente estudo, no entanto correlações não foram encontradas.

De qualquer forma, o fato de o presente estudo ter priorizado uma escola municipal que atende a população de crianças residentes em uma região periférica e vulnerável do município pode ajudar a compreender o resultado obtido em relação à percentagem de problemas de saúde mental infantil. Não obstante, o índice encontrado ainda é substancialmente maior se comparado ao grupo menos favorecido economicamente do estudo de Fleitlich e Goodman (2001). Estudos comparativos, envolvendo participantes de outras regiões do município poderiam contribuir na compreensão desse resultado.

Outra hipótese se refere ao instrumento utilizado, o SDQ, que possui três versões destinadas a três tipos de respondentes, sendo que este estudo utilizou somente a versão para pais e/ou responsáveis, ou seja, a saúde mental infantil foi avaliada a partir de uma única ótica, o que indica uma limitação do estudo.

Quanto às práticas de cuidado dos pais com as crianças, as análises indicaram relação entre o responsável brincar e dar broncas mesmo sem motivos e as escalas de problemas de saúde mental.

Os achados da literatura reforçam os resultados encontrados sobre o brincar, uma vez que o brincar deve ser incentivado pelos pais, pois a brincadeira estimula o bom desempenho escolar, além da aquisição de algumas habilidades como a concentração e a autoconfiança (SOARES et al., 2004). É possível que em contextos de vulnerabilidade tais práticas tenham e assumam ainda um papel e valor que ultrapassam apenas ganhos de habilidades específicas, possibilitando momentos de envolvimento e afeto entre cuidador e criança.

Em relação às regras e responsabilidades na família, o estudo apontou que nas famílias onde as crianças possuem regras e responsabilidades há menor frequência de problemas de conduta. Esse achado é reforçado pelo estudo de Ferriolli et al. (2007), uma vez que este também identificou que em famílias que possuem rotina diária com horários e uma estrutura adequada para lição de casa, as crianças apresentavam menos problemas de saúde mental.

Assim, o brincar e a existência de regras e responsabilidades na família parecem estar relacionados à práticas do contexto familiar que podem proteger ou amenizar conseqüências na saúde mental das crianças, na presença de risco, como a situação socioeconômica desfavorável, por exemplo. Estudos que investiguem estas variáveis em uma amostra maior são fundamentais para a investigação dessa hipótese.

O desempenho da criança na escola esteve correlacionado com hiperatividade e comportamento pró-social. Estudos reforçam este resultado uma vez que apontam que as crianças com dificuldades sócio-emocionais apresentam maiores riscos para desenvolver dificuldades acadêmicas seguindo para o abandono escolar (FLEITLICH, 2005). Além disso, o fracasso escolar está intimamente ligado à situação emocional das crianças (BASTOS; ALMEIDAFILHO,1988).

Autores apontam para a importância do auxílio dos pais nas atividades escolares das crianças, uma vez que o suporte para a realização escolar se concretiza por meio do envolvimento direto dos pais com a vida acadêmica dos filhos. São exemplos dessa modalidade de suporte a disposição de tempo e espaço adequado em casa para a realização dos deveres escolares, a exigência de cumprimento desses deveres, o intercâmbio regular com o professor e uma rotina de horários para as atividades diárias básicas (D'AVILA et al., 2005).

Nesta medida, sugere-se a implementação e efetivação de canais de diálogo e intercâmbios com a escola e professores, no sentido de esclarecer e debater sobre o papel dos mesmos, como fatores protetivos no desenvolvimento da saúde mental dessas crianças.

Não obstante, importa, aqui, destacar a condição de risco que agrega fatores, ou seja, apresentar uma situação econômica desfavorável, dificuldades no desenvolvimento sócioemocional e dificuldades escolares caracterizam uma condição de vulnerabilidade vivenciada pelas crianças. Assim, a análise dos fatores que favoreçam ou não o desenvolvimento de problemas relativos à saúde mental deve considerar os outros aspectos (individuais, familiares, contextuais, sócioculturais) que potencialmente interferem nas condições de vida e de desenvolvimento de crianças e famílias.

É necessário apontar que este estudo apresentou algumas limitações devido ao número reduzido de participantes, o que não permite generalizações para toda a população. Porém as correlações existentes e as diferenças encontradas em relação aos outros estudos são indicativos importantes que podem ser aprofundados, visto que na 
literatura são raros os estudos que abordam a saúde mental infantil, o cotidiano e contexto familiar.

Assim, considera-se que este estudo contribui para o conhecimento sobre fatores de risco e proteção a saúde mental infantil em relação ao contexto familiar e práticas de cuidado de famílias em condições sócio econômicas muito desfavorecidas. Além disso, vem acrescentar elementos para a discussão sobre as intervenções e implantações de serviços de prevenção e políticas públicas voltados à saúde mental infantil.

Destaca-se por fim a relevância da necessidade de implementação de políticas e serviços de saúde mental e práticas preventivas nesta área, voltados para a população de crianças e jovens. Tal necessidade é também reforçada por estudiosos que apontam que se houver investimento na saúde mental infantil as repercussões serão imediatas, pois haverá diminuição do sofrimento dessa população e dos familiares. A longo prazo, favorecerão a diminuição da criminalidade, abuso de substância, fracasso e abandono escolar, desenvolvimento de transtornos de personalidade e transtornos mentais na vida adulta e capacitar o desenvolvimento desses jovens para que eles possam atuar futuramente como pais (FLEITLICH; GOODMAN, 2002; FLEITLICH, 2005).

\section{CONCLUSÃO}

Considera-se que o estudo atingiu os objetivos propostos, contribuindo para melhor conhecimento dos fatores de risco e proteção associados à saúde mental infantil. Consequentemente os resultados encontrados podem subsidiar o planejamento e implementação de políticas públicas no campo da saúde mental infantil, assim como apresentar elementos para reflexões e para a compreensão dos processos e indicadores familiares envolvidos nessa condição.

Dessa forma, as informações obtidas podem contribuir para o debate acerca de políticas públicas e programas efetivos para atender a todas e crianças e adolescentes que necessitam de atenção à saúde mental.

MATSUKURA, T. S.; FERNANDES, A. D. S. A.; CID, M. F. B. Risk and protective factors for mental health in children: the family context. Rev. Ter. Ocup. Univ. São Paulo, v. 23, n. 2, p. 122129, maio/ago. 2012.

\begin{abstract}
Some family characteristics can bring risks for the healthy development of children, among them stand out, low income, low parental education, high levels of family stress. Together with risk factors that are subject to the children, there are the protective factors that can induce human development in the presence of risk. The aim of this study was to identify associations between child mental health and variables considered of risk or protection present in the familiar context of elementary school children. The research was developed with the sample of 39 guardians of children from 1 st to 5 th year of elementary school, in a public school located on the outskirts of São Carlos-SP. The instruments used were: Strengths and Difficulties questionnaire (SDQ), Critério de Classificação Econômica Brasil (CCEB) and the Questionário de atividades cotidianas (QAC). The statistical analysis was descriptive, correlational and with the comparison between the groups, using nonparametric MannWhitney and Kendall's. The outcomes showed that the variable to "scolding for no reason" and "guardian plays with the child" is related with the presence of mental health problems. In contrast, the variable "rules and responsibilities in the family" was related to higher levels of child mental health.
\end{abstract}

KEY WORDS: Mental health; Family/psychology;Child; Risk factors; Child psychology/ manpower.

\title{
REFERÊNCIAS
}

ABEP - Associação Brasileira de Empresas de Pesquisa. Disponível em: $<$ http://www.abep.org.br>.

ASSIS, S. G.; AVANCI, J. Q.; OLIVEIRA, R. V. C. Desigualdades socioeconômicas e saúde mental infantil. Rev. Saúde Pública, São Paulo, v. 43, supl. 1, p. 92-100, 2009.

BASTOS, A. C.; ALMEIDA-FILHO, N. Determinação social da saúde mental infantil: revisão da literatura epidemiológica. Psicologia: Teoria Pesq., v. 4, n. 3, p. 268-282, 1988.

CAMPOS, S. E. A.; et al. O lazer cotidiano do Jardim Gonzaga - São Carlos. In: ENCONTRO NACIONAL DE RECREAÇÃO E LAZER, 15. SANTO ANDRÉ, SP, 2003. Lazer e trabalho: novos significados na sociedade contemporânea. Anais... Santo André, 2003. 
CID, M. F. B.; MATSUKURA, T. S. Mães com transtorno mental e seus filhos: risco e desenvolvimento. Mundo Saúde. v. 34, n. 1, p 73-81. 2010.

CID, M. F. B.; MATSUKURA, T. S. Prevalência de problemas de saúde mental infantil: Identificação de fatores de risco e proteção. 2009. Trabalho de doutorado em andamento. Programa de pósgraduação em Educação Especial da Universidade Federal de São Carlos, 2009.

COIE, J. D.; et al. The science of prevention: a conceptual framework and some directions for a national research program. Am. Psychol., v. 48, n. 10, p. 1013-1022, 1993.

CURY, C. R.; GOLFETO, J. H. Strengths and difficulties questionnaire (SDQ): a study of school in Ribeirão Preto. Rev. Bras. Psiquiatr, v. 25, n. 3, 2003.

D'AVILA-BACARJI, K. M. G.; MARTURANO, E. M.; ELIAS, L. C. S. Suporte parental: Um estudo sobre crianças com queixas escolares. Psicologia Estudo, v. 10, p. 107-115, 2005.

DEKOVIC, M. Risk and protective factors in the development of problem behavior during adolescence. J. Youth Adolesc., v. 28, n. 6, p. 667-685, 1999.

FERRIOLI, S. H. T.; MARTURANO, E. M.; PUNTEL, L. P. Contexto familiar e problemas de saúde mental infantil no Programa Saúde da Família. Rev. Saúde Pública, v. 41, n. 2, 2007.

FLEITLICH, B. A saúde nos jovens brasileiros: quais são os principais problemas e como podemos ajudar? J. Bras. Psiquiatr., v. 54, n. 3, p. 168-169, 2005.

FLEITLICH, B.; GOODMAN, R. Epidemiologia. Rev. Bras. Psiquiatr., v. 22, n. 2, p. 2-6, 2000.

FLEITLICH, B.; GOODMAN, R. Social factors associated with child menatl health problems in Brasil: cross sectional survey. $\mathrm{Br}$. Med. J., v. 323, p. 599, 2001.

FLEITLICH, B.; GOODMAN, R. Implantação e implementação de serviços de saúde mental comunitários para crianças e adolescentes. Rev. Bras. Psiquiatr., v. 24, n. 1, 2002.

FLEITLICH, B.; GOODMAN, R. Prevalence of child and adolescent psychiatric disorders in southeast Brasil. J. Am. Acad. Child Adolesc. Psychiatry, v. 42, n. 6, p. 728-734, 2004.

GREENBERG, M. T.; DOMITROVICH, C.; BUMBARGER, B. The Prevention of Mental Disorders in school-aged children: current state of the field. Prevent. Treatment, v. 4, n. 1, 2001.
HALPERN, R.; FIGUEIRAS, A. C. M. Influências ambientais na saúde mental da criança. J. Pediatria, Porto Alegre, v. 80, n. 2, p. 104-110, 2004.

MONDIN, E. M. C. Um olhar ecológico da família sobre o desenvolvimento humano. Psicol. Argumento, Curitiba, v. 23, n. 41 p. 25-35, 2005.

ORGANIZAÇÃO MUNDIAL DA SAÚDE. Strengthening mental health promotion. Geneva, World Health Organization, 2001. (Fact sheet no.220).

PACHECO, J.; et al. Estabilidade do comportamento anti-social na transição da infância para a adolescência: Uma perspectiva desenvolvimentista. Psicol. Reflexão Critica, v. 18, n. 1, p. 5561,2005

PAULA, C. S.; MIRANDA, C. T.; BORDIN, I. A. L. S. Saúde mental na infância e adolescência: revisão dos estudos epidemiológicos brasileiros. In: LAURIDSEN, E.; RIBEIRO; O. Y.; TANAKA (Orgs.). Atenção em saúde mental para crianças e adolescentes no SUS. São Paulo: Hucitec, 2010.

PAULA, C. S.; DUARTE, C. S.; BORDIN, I. S. Prevalence of mental health problems in children and adolescents from the outskirts of São Paulo City: treatment needs and service capacity evaluation. Rev. Bras. Psiquiatr., v. 29, n. 1, 2006/2007.

PATEL, V.; et al. Mental health of young people: a global publichealth challenge. Lancet, v. 369, 2007.

PUNTEL, L. P. Problemas emocionais e de comportamento em crianças de 6 a 12 anos cadastradas em um Núcleo de Atenção Primária e Saúde da Família. 2005. Dissertação de Mestrado Faculdade de Medicina de Ribeirão Preto, Universidade de São Paulo, 2005.

SANTOS, L. C.; MARTURANO, E. M. Crianças com dificuldades de aprendizagem: um estudo de segmento. Psicol. Reflexão Crítica, v. 12 , p. 377-394, 1999.

SCHWENGBER, D. D. S.; PICCININI, C. A. O impacto da depressão pós-parto para a interação mãe-bebê. Estudos Psicol., v. 8, n. 3, p. 403-411, 2003.

SOARES, M. R. Z.; SOUZA, S. R.; MARINHO, M. L. Envolvimento dos pais: incentivo à habilidade de estudo em crianças. Estudos Psicol., Maringá, v. 21, n. 3, p. 253-260, 2004.

VITOLO, Y. L. C.; Fleitlich-Bilyk, B.; Goodman, R.; Bordin, I. A. S. Crenças e atitudes educativas dos pais e problemas de saúde mental em escolares. Rev. Saúde Pública, v. 39, n. 5, p. 716-724, 2005. 